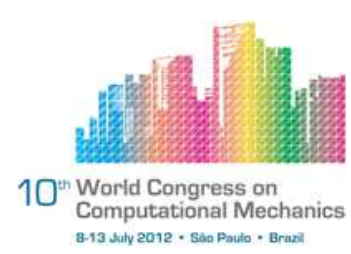

\title{
STATIC AND DYNAMIC ANALYSIS OF A THIN-WALLED LAYERED CYLINDER BY REFINED 1D THEORIES
}

\author{
A. Varello ${ }^{1}$, E. Carrera ${ }^{1}$ \\ ${ }^{1}$ Department of Mechanical and Aerospace Engineering (alberto.varello@ polito.it)
}

\begin{abstract}
This paper presents an application of refined one-dimensional (1D) models to the static and free vibration analysis of thin-walled layered structures. Carrera Unified Formulation (CUF) is employed to easily introduce higher-order 1D models with a variable order of expansion for the displacement unknowns over the beam cross-section. Classical Euler-Bernoulli beam theory is obtained as a particular case of these variable kinematic models while a higher-order expansion permits the detection of the in-plane cross-section deformation. Finite element (FE) method is used to provide numerical results. In particular, the case of a deformable clamped-clamped thin-walled layered cylinder loaded by a non-uniform internal pressure is discussed. The static analysis reveals the model capabilities in accurately describing the three-dimensional deformation of the cylinder. The free vibration analysis provides results not detectable by typical $1 D$ models in excellent agreement with a solid (3D) FE solution. The present models do not introduce additional numerical problems with respect to classical beam theories. Moreover, the results clearly show that finite elements which are formulated in the CUF framework offer shell-type capabilities in analyzing thin-walled structures with a remarkable reduction in the computational cost required.
\end{abstract}

Keywords: 1D Refined Models, Unified Formulation, Higher-order, Finite Elements.

\section{INTRODUCTION}

In many areas such as aerospace, civil and biomechanical engineering different kinds of slender structures are involved nowadays. Typical examples of these one-dimensional (1D) structures are for instance rotor and wind blades, aircraft wings, bridges and towers, and even veins. Such beam-like components can be analyzed via 1D formulations and one main advantage is that $1 \mathrm{D}$ models require a lower computational cost compared with $2 \mathrm{D}$ plate and shell or 3D solid models [1].

The 1D models used in early studies of slender structures were based on classical theories, such as Euler-Bernoulli [13] and Timoshenko [30] theories. The growing use of advanced composite and sandwich materials in thin-walled beam-like structures has revealed that 1D theories have to be refined in order to predict the behavior of such complex structures in an accurate way. In last decades, many theoretical and computational approaches were taken to address issues such as warping effects and in-plane cross-secton deformation. Recently, 
refined theories such as those based on the 1D Carrera Unified Formulation (CUF) [7, 8] and variational asymptotic methods (VABS) [32] as well as the Generalized Beam Theory (GBT) [25] have presented remarkable advances in static, buckling, and free vibration analysis.

A detailed review of several theories for vibrations and wave propagation was presented by Kapania and Raciti [20]. A first formulation of a second-order theory by using crosssectional warping and transverse direct stress was proposed by Stephen and Levinson [28]. Heyliger and Reddy [16] proposed a third-order theory with a quadratic variation of the shear strain accross the cross-section. Its refined version, with transverse normal stress components, was reported later by Soldatos and Elishakoff [27]. Early fourth-order beam theories were formulated by Levinson [21], Rychter [24] and were extended by Bickford [2] to the dynamic analysis. Kant and Gupta [18] proposed a refined FE higher-order model with quadratic transverse shear strain that was applied to the free vibration analysis of angle-ply laminated, deep sandwich and composite beams [22, 23]. Kant et al.[19] provided an analytical solution to the natural frequency analysis of thick and thin composite beams by accurately desribing the cross-section warping. The formulation of two higher-order shear deformation theories by Subramanian [29] satisfied the traction-free surface conditions at the top and bottom beam

surfaces. A higher-order FE model based on classical laminated theory presented higherfrequencies analysis capabilities for the vibration response of laminated tapered beams [14, 15]. Recently, Şimşek and Kocatürk [26] highlighted that a third-order shear deformation theory gives significantly better results than classical theories in the case of short beams and high mode numbers.

As mentioned, higher-order 1D models with generalized displacement variables based on CUF have recently been proposed by Carrera and co-authors for the analysis of isotropic [8] and composite structures [10, 31]. CUF has been developed over the last decade for plate/shell models [6,3] and it has recently been extended to static and dynamic 1D modeling [7, 9, 12]. CUF is a hierarchical formulation which considers the order of the model as a free-parameter of the analysis. In other words, models of any order can be obtained with no need for ad hoc formulations by exploiting a systematic procedure. Euler-Bernoulli and Timoshenko beam theories are obtained as particular cases of the first-order expansion.

In this paper, an application of CUF one-dimensional models to the static and free vibration analysis of thin-walled structures is presented. The finite element method (FEM) is used to handle arbitrary geometries and loading conditions. At first, a static case is considered and comparisons with solid FE solutions are made in order to assess the present 1D model and enhance its accuracy. The influence of higher-order effects over the three-dimensional cross-section deformation, not detectable by classicalbeam theories, is enhanced also on the evaluation of vibrational modes of layered structures. The use of variable kinematic 1D CUF models reveals their shell-type capabilities in accurately describing the dynamic behavior of thin-walled structures.

\section{PRELIMINARIES}

A slender structure with axial length $L$ is considered and studied as a beam. The intersection of the beam with a plane that is perpendicular to its axis identifies the beam's cross-section $\Omega$. A global cartesian coordinate system composed of $x$ and $z$ axes parallel to the 
cross-section plane is defined, whereas $y$ represents the out-of-plane coordinate. However, the $y$ axis is not necessarily a centroidal one. The choice of the cross-section is arbitrary, since it does not affect the following theoretical formulation. In the present work, the thin layered annular cross-section shown in Fig. 1 is considered. The three layers are denoted as 1, 2, and 3. Therefore, a thin-walled layered cylinder represents the structure to be studied.

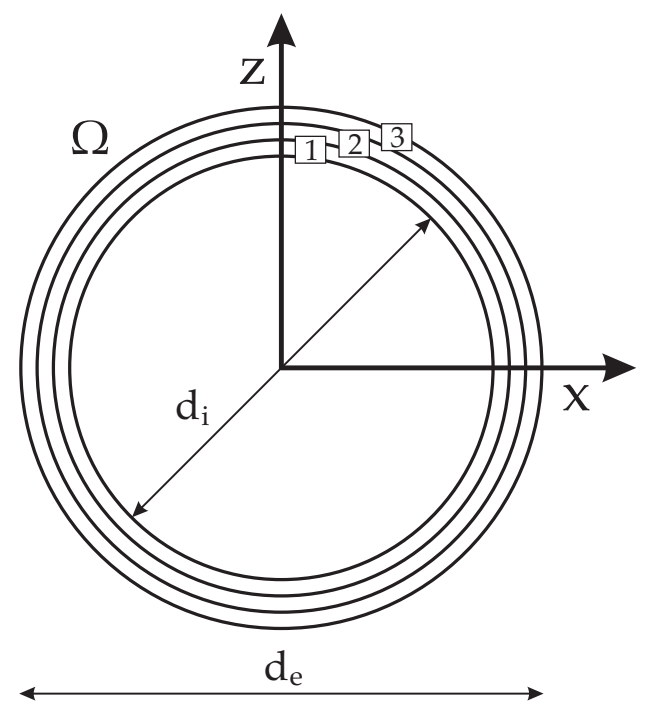

Figure 1. Geometry of the cylinder cross-section.

The cartesian components of the displacement vector $\boldsymbol{u}(x, y, z)$ are $u_{x}, u_{y}$, and $u_{z}$. The stress $\sigma$ and the strain $\varepsilon$ are grouped in vectors as follows:

$$
\begin{array}{lll}
\boldsymbol{\sigma}_{p}=\left\{\begin{array}{lll}
\sigma_{z z} & \sigma_{x x} & \sigma_{z x}
\end{array}\right\}^{T} & \boldsymbol{\varepsilon}_{p}=\left\{\begin{array}{llll}
\varepsilon_{z z} & \varepsilon_{x x} & \varepsilon_{z x}
\end{array}\right\}^{T} \\
\boldsymbol{\sigma}_{n}=\left\{\begin{array}{llll}
\sigma_{z y} & \sigma_{x y} & \sigma_{y y}
\end{array}\right\}^{T} & \boldsymbol{\varepsilon}_{n}=\left\{\begin{array}{lll}
\varepsilon_{z y} & \varepsilon_{x y} & \varepsilon_{y y}
\end{array}\right\}^{T}
\end{array}
$$

where superscript $T$ stands for the transposition operator. Subcript $n$ refers to quantities related to the beam cross-section $\Omega$, whereas subscript $p$ refers to quantities related to the out-of-plane direction. In the case of small displacements with respect to the length $L$, the linear relations between strain and displacement components hold and a compact vectorial notation can be adopted:

$$
\begin{aligned}
& \boldsymbol{\varepsilon}_{p}=\boldsymbol{D}_{p} \boldsymbol{u} \\
& \boldsymbol{\varepsilon}_{n}=\boldsymbol{D}_{n} \boldsymbol{u}=\boldsymbol{D}_{n p} \boldsymbol{u}+\boldsymbol{D}_{n y} \boldsymbol{u}
\end{aligned}
$$

where $\boldsymbol{D}_{p}, \boldsymbol{D}_{n p}$, and $\boldsymbol{D}_{n y}$ are differential matrix operators:

$$
\boldsymbol{D}_{p}=\left[\begin{array}{ccc}
0 & 0 & \frac{\partial}{\partial z} \\
\frac{\partial}{\partial x} & 0 & 0 \\
\frac{\partial}{\partial z} & 0 & \frac{\partial}{\partial x}
\end{array}\right], \quad \boldsymbol{D}_{n p}=\left[\begin{array}{ccc}
0 & \frac{\partial}{\partial z} & 0 \\
0 & \frac{\partial}{\partial x} & 0 \\
0 & 0 & 0
\end{array}\right], \quad \boldsymbol{D}_{n y}=\left[\begin{array}{ccc}
0 & 0 & \frac{\partial}{\partial y} \\
\frac{\partial}{\partial y} & 0 & 0 \\
0 & \frac{\partial}{\partial y} & 0
\end{array}\right]
$$

According to Eq. 1, the generalized Hooke's law for isotropic materials is:

$$
\begin{aligned}
& \boldsymbol{\sigma}_{p}=\boldsymbol{C}_{p p} \varepsilon_{p}+\boldsymbol{C}_{p n} \varepsilon_{n} \\
& \boldsymbol{\sigma}_{n}=C_{n p} \varepsilon_{p}+C_{n n} \varepsilon_{n}
\end{aligned}
$$


where matrices $C_{p p}, C_{p n}, C_{n p}$ and $C_{n n}$ are:

$$
\begin{aligned}
\boldsymbol{C}_{p p} & =\left[\begin{array}{ccc}
C_{11} & C_{12} & 0 \\
C_{12} & C_{22} & 0 \\
0 & 0 & C_{44}
\end{array}\right], \quad \boldsymbol{C}_{p n}=\boldsymbol{C}_{n p}^{T}=\left[\begin{array}{ccc}
0 & 0 & C_{13} \\
0 & 0 & C_{23} \\
0 & 0 & 0
\end{array}\right], \\
\boldsymbol{C}_{n n} & =\left[\begin{array}{ccc}
C_{55} & 0 & 0 \\
0 & C_{66} & 0 \\
0 & 0 & C_{33}
\end{array}\right],
\end{aligned}
$$

For the sake of brevity, the dependence of the coefficients $C_{i j}$ on Young's modulus, Poisson's ratios, and shear modulus is not reported here. It can be found in Jones [17]. In this paper isotropic materials are considered. The extension to composite beams will be presented in future papers.

\section{VARIABLE KINEMATIC 1D MODELS}

According to the framework of Carrera Unified Formulation (CUF) [8], the displacement field is assumed to be an expansion of a certain class of functions $F_{\tau}$, which depend on the cross-section coordinates $x$ and $z$ :

$$
\boldsymbol{u}(x, y, z, t)=F_{\tau}(x, z) \boldsymbol{u}_{\tau}(y, t) \quad \tau=1,2, \ldots, N_{u}=N_{u}(N)
$$

The compact expression is based on Einstein's notation: repeated subscript $\tau$ indicates summation. The number of expansion terms $N_{u}$ depends on the expansion order $N$, which is a free parameter of the formulation. Taylor's polynomials are chosen as cross-section functions $F_{\tau}$. Most displacement-based theories can be formulated on the basis of the above generic kinematic field. For instance, when $N=2$, the second-order axiomatic displacement field is given by:

$$
\begin{aligned}
& u_{x}=u_{x 1}+u_{x 2} x+u_{x 3} z+u_{x 4} x^{2}+u_{x 5} x z+u_{x 6} z^{2} \\
& u_{y}=u_{y 1}+u_{y 2} x+u_{y 3} z+u_{y 4} x^{2}+u_{y 5} x z+u_{y 6} z^{2} \\
& u_{z}=u_{z 1}+u_{z 2} x+u_{z 3} z+u_{z 4} x^{2}+u_{z 5} x z+u_{z 6} z^{2}
\end{aligned}
$$

Subsequently, the classical beam models such as Euler-Bernoulli's (EBBM) [13] and Timoshenko's (TBM) [30] are easily derived from the first-order approximation model. Timoshenko beam model (TBM) can be obtained by setting terms $\left\{u_{i j}: i=x, z ; j=2,3\right\}$ equal to zero. In addition, an infinite rigidity in the transverse shear is also adopted for EBBM by penalizating $\varepsilon_{x y}$ and $\varepsilon_{y z}$ via a high penalty value in the following constitutive equations:

$$
\sigma_{y z}=C_{55} \varepsilon_{y z} \quad \sigma_{x y}=C_{66} \varepsilon_{x y}
$$

Higher-order models provide an accurate description of the shear mechanics, the cross-section deformation, Poisson's effect along the spatial directions and the torsional mechanics in more detail than classical models do. EBBM neglects them all, since it was formulated to describe the bending mechanics. TBM takes into account constant shear stress and strain components. Classical theories and first-order models require the assumption of opportunely reduced material stiffness coefficients to correct Poisson's locking effect [4, 5]. According to Carrera and Giunta [7], the same technique is used here to correct Poisson's locking. 


\section{FINITE ELEMENT FORMULATION}

Following standard FEM, the unknown variables in the element domain are expressed in terms of their values corresponding to the element nodes [8]. For the sake of completeness, some details about the formulation of CUF finite elements are here retrieved from previous works $[11,12]$ and extended to the dynamic response analysis. By introducing the shape functions $N_{i}$ and the nodal displacement vector $\boldsymbol{q}$, the displacement field becomes:

$$
\boldsymbol{u}(x, y, z, t)=F_{\tau}(x, z) N_{i}(y) \boldsymbol{q}_{\tau i}(t) \quad i=1,2, \ldots, N_{N}
$$

where:

$$
\boldsymbol{q}_{\tau i}=\left\{\begin{array}{lll}
q_{u_{x \tau i}} & q_{u_{y_{\tau i}}} & q_{u_{z_{\tau i}}}
\end{array}\right\}^{T}
$$

contains the degrees of freedom of the $\tau^{\text {th }}$ expansion term corresponding to the $i^{\text {th }}$ element node. One-dimensional elements with 4 nodes are formulated. Third-order Lagrange polynomials are used as shape functions. For the sake of brevity, more details are not reported here, but can be found in Carrera et al.[9, 11]. This beam model can be easily extended to mixed theories. However, this work presents a displacement-based formulation. The variational statement is therefore the Principle of Virtual Displacements:

$$
\delta L_{i n t}=\int_{V}\left(\delta \boldsymbol{\varepsilon}_{n}^{T} \boldsymbol{\sigma}_{n}+\delta \boldsymbol{\varepsilon}_{p}^{T} \boldsymbol{\sigma}_{p}\right) d V=\delta L_{e x t}-\delta L_{i n e}
$$

where $L_{i n t}$ is the internal strain energy, $L_{\text {ext }}$ is the work of external loadings, and $L_{\text {ine }}$ is the work of inertial loadings. $\delta$ stands for the virtual variation. The damping effect is neglected for the sake of simplicity. Substituting Eq. 9 into Eq. 2 and using the fact that $F_{\tau}$ are independent of $y$, the strain vectors can be written as:

$$
\begin{aligned}
& \boldsymbol{\varepsilon}_{n}=\left(\boldsymbol{D}_{n p} F_{\tau} \boldsymbol{I}\right) N_{i} \boldsymbol{q}_{\tau i}+F_{\tau}\left(\boldsymbol{D}_{n y} N_{i} \boldsymbol{I}\right) \boldsymbol{q}_{\tau i} \\
& \boldsymbol{\varepsilon}_{p}=\left(\boldsymbol{D}_{p} F_{\tau} \boldsymbol{I}\right) N_{i} \boldsymbol{q}_{\tau i}
\end{aligned}
$$

where $\boldsymbol{I}$ is the identity matrix. The expression of the internal strain energy (Eq. 11) can be rewritten in terms of virtual nodal displacements as follows:

$$
\delta L_{i n t}=\delta \boldsymbol{q}_{\tau i}^{T} \boldsymbol{K}^{i j \tau s} \boldsymbol{q}_{s j}
$$

where Eq. 4 has been used. The $3 \times 3$ fundamental nucleus of the structural stiffness matrix presented in Eq. 13 can be shown to have the following explicit equation:

$$
\begin{aligned}
& \boldsymbol{K}^{i j \tau s}=E_{i j} \triangleleft\left(\boldsymbol{D}_{n p}^{T} F_{\tau} \boldsymbol{I}\right)\left[\boldsymbol{C}_{n p}\left(\boldsymbol{D}_{p} F_{s} \boldsymbol{I}\right)+\boldsymbol{C}_{n n}\left(\boldsymbol{D}_{n p} F_{s} \boldsymbol{I}\right)\right]+ \\
& \left(\boldsymbol{D}_{p}^{T} F_{\tau} \boldsymbol{I}\right)\left[\boldsymbol{C}_{p p}\left(\boldsymbol{D}_{p} F_{s} \boldsymbol{I}\right)+\boldsymbol{C}_{p n}\left(\boldsymbol{D}_{n p} F_{s} \boldsymbol{I}\right)\right] \triangleright_{\Omega}+ \\
& E_{i j, y} \triangleleft\left[\left(\boldsymbol{D}_{n p}^{T} F_{\tau} \boldsymbol{I}\right) \boldsymbol{C}_{n n}+\left(\boldsymbol{D}_{p}^{T} F_{\tau} \boldsymbol{I}\right) \boldsymbol{C}_{p n}\right] F_{s} \triangleright_{\Omega} \boldsymbol{I}_{\Omega y}+ \\
& E_{i, y j} \boldsymbol{I}_{\Omega y}^{T} \triangleleft F_{\tau}\left[\boldsymbol{C}_{n p}\left(\boldsymbol{D}_{p} F_{s} \boldsymbol{I}\right)+\boldsymbol{C}_{n n}\left(\boldsymbol{D}_{n p} F_{s} \boldsymbol{I}\right)\right] \triangleright_{\Omega}+ \\
& E_{i, y} j_{, y} \boldsymbol{I}_{\Omega y}^{T} \triangleleft F_{\tau} \boldsymbol{C}_{n n} F_{s} \triangleright_{\Omega} \boldsymbol{I}_{\Omega y}
\end{aligned}
$$


where:

$$
\begin{gathered}
\boldsymbol{I}_{\Omega y}=\left[\begin{array}{ccc}
0 & 0 & 1 \\
1 & 0 & 0 \\
0 & 1 & 0
\end{array}\right] \quad \triangleleft \ldots \triangleright_{\Omega}=\int_{\Omega} \ldots d \Omega \\
\left(E_{i j}, E_{\left.i j_{y}, E_{i, y}, E_{i, y j}\right)}\right)=\int_{l}\left(N_{i} N_{j}, N_{i} N_{j, y}, N_{i, y} N_{j}, N_{i_{y}} N_{j, y}\right) d y
\end{gathered}
$$

The symbol $\triangleleft \ldots \triangleright_{\Omega}$ indicates integration over the cross-section. In the case that the beam cross-section consists of subsections with different laminations as in the present work, the matrices $C_{p p}, C_{p n}, C_{n p}$ and $C_{n n}$ are different for each subsection. Hence, it is important to note that such the integration over the cross-section has to include all the subsections contributions.

The virtual variation of the work of inertial loadings is:

$$
\delta L_{i n e}=\int_{V} \boldsymbol{u}^{T} \rho \ddot{\boldsymbol{u}} d V
$$

where $\rho$ is the density of the material and $\ddot{\boldsymbol{u}}$ is the acceleration vector. By retrieving Eq. 9, $\delta L_{\text {ine }}$ can be rewritten in terms of virtual nodal displacements as follows:

$$
\delta L_{i n e}=\delta \boldsymbol{q}_{\tau i}^{T} \int_{\Omega} \rho\left(F_{\tau} \boldsymbol{I}\right)\left[\int_{l} N_{i} N_{j} d y\right]\left(F_{s} \boldsymbol{I}\right) d \Omega \ddot{\boldsymbol{q}}_{s j}
$$

where $\ddot{q}$ is the nodal acceleration vector. The virtual variation of the work of inertial loadings can be finally expressed in the following compact notation:

$$
\delta L_{i n e}=\delta \boldsymbol{q}_{\tau i}^{T}\left[\rho E_{i j} \triangleleft F_{\tau} F_{s} \triangleright_{\Omega} \boldsymbol{I}\right] \ddot{\boldsymbol{q}}_{s j}=\delta \boldsymbol{q}_{\tau i}^{T} \boldsymbol{M}^{i j \tau s} \ddot{\boldsymbol{q}}_{s j}
$$

The $3 \times 3$ fundamental nucleus of the mass matrix presented in Eq. 19 is therefore a diagonal matrix. The virtual work of external loads variationally consistent with the above method is here derived for the case of a generic concentrated load $\boldsymbol{P}=\left\{P_{u_{x}} P_{u_{y}} P_{u_{z}}\right\}^{T}$ acting on the load application point $\left(x_{P}, y_{P}, z_{P}\right)$. By using Eq. $9, \delta L_{e x t}$ becomes:

$$
\delta L_{e x t}=\delta \boldsymbol{u}^{T} \boldsymbol{P}=\delta \boldsymbol{q}_{\tau i}^{T} F_{\tau} N_{i} \boldsymbol{P}=\delta \boldsymbol{q}_{\tau i}^{T} \boldsymbol{F}_{\tau i}
$$

where $F_{\tau}$ is evaluated in $\left(x_{P}, z_{P}\right)$ and $N_{i}$ is calculated in $y_{P}$. Any other loading condition can be similarly treated. From Eq.s 11, 13, 19, and 20 the governing equation of motion can be derived through a finite element assembly procedure:

$$
M \ddot{q}+K \boldsymbol{q}=\boldsymbol{F}
$$

where $\boldsymbol{M}$ is the mass matrix, $\boldsymbol{K}$ is the stiffness matrix and $\boldsymbol{F}$ is the vector of equivalent nodal forces. It should be noted that no assumptions on the expansion order have been made so far. Therefore, it is possible to obtain variable kinematic 1D models without changing the formal expression of the nucleus components. Thanks to the CUF, the present model is invariant with respect to the order of the beam theory and the type of element used in the finite element axial discretization. Shear locking is corrected through selective integration [1]. 
In conclusion, the static structural response is computed as solution of the following equation, which is a particular form of Eq. 23 by neglecting the inertial contribution:

$$
\boldsymbol{K} \boldsymbol{q}=\boldsymbol{F}
$$

The free vibration analysis of the structure is carried out by considering the homogeneous case of Eq. 23 by neglecting the contribution of external loadings:

$$
\boldsymbol{M} \ddot{\boldsymbol{q}}+\boldsymbol{K} \boldsymbol{q}=0
$$

Introducing harmonic solutions, it is possible to compute the natural angular frequencies, $\omega_{k}$, and the natural frequencies, $f_{k}$, by solving an eigenvalue problem:

$$
\left[-\omega_{k}^{2} \boldsymbol{M}+\boldsymbol{K}\right] \boldsymbol{q}_{k}=0
$$

where $\boldsymbol{q}_{k}$ is the $k^{\text {th }}$ eigenvector.

\section{RESULTS AND DISCUSSION}

As previously mentioned, the structure studied in the present paper is a three-layer cylinder. The layers are made of different isotropic materials. The material and geometrical properties of the layers are summarized in Table 1. The thickness $t$ is equal for each layer and is small enough to consider overall the cylinder as a thin-walled structure, since the external and internal diameter are respectively equal to $d_{e}=100 \mathrm{~mm}$ and $d_{i}=94 \mathrm{~mm}$. The length $L$ of the cylinder is equal to $500 \mathrm{~mm}$. For all the following analysis, a clamped boundary condition is taken into account for both the free edges (at $y=0$ and $y=L$ ).

Table 1. Material and geometrical properties of the cylinder layers.

\begin{tabular}{lrrr}
\hline \hline Property & Layer 1 & Layer 2 & Layer 3 \\
\hline \hline $\mathrm{t}[\mathrm{mm}]$ & 1 & 1 & 1 \\
$\mathrm{E}[\mathrm{GPa}]$ & 69 & 30 & 15 \\
$\nu$ & 0.33 & 0.33 & 0.33 \\
$\rho\left[\mathrm{kg} / \mathrm{m}^{3}\right]$ & 2700 & 2000 & 1800 \\
\hline
\end{tabular}

In order to easily present the deformation of the cylinder, a cylindrical coordinate system $r-\theta-y$ is now introduced. The plane $r-\theta$ is the cross-section plane. The $r$ coordinate goes along the radial direction, whereas the $\theta$ coordinate is an angle measured counterclockwise from the axis $-z$. For the sake of simplicity, the origin of the cylindrical system overlaps the cross-section center of mass as well as the origin of the global coordinate system.

\subsection{Static analysis}

A uniform pressure $p=14.8 \mathrm{MPa}$ is applied on the internal edge of the cylinder as shown in Fig. 2. In particular, the loading is applied along all the length of the structure 
and only on the upper side of layer $1\left(r=d_{i} / 2,90^{\circ} \leq \theta \leq 270^{\circ}, 0 \leq y \leq L\right)$. Although the structure here analyzed is axisymmetric, its deformed configuration is not expected to be axisymmetric due to the loading distibution. Nevertheless, the solution will be symmetrical with respect to both $x=0$ and $y=L / 2$ planes. Given the solution's simmetry with respect to $y=L / 2$ plane, the maximum deformation is placed on the section lying on this plane. This section is denoted as mid-span section from this point forward.

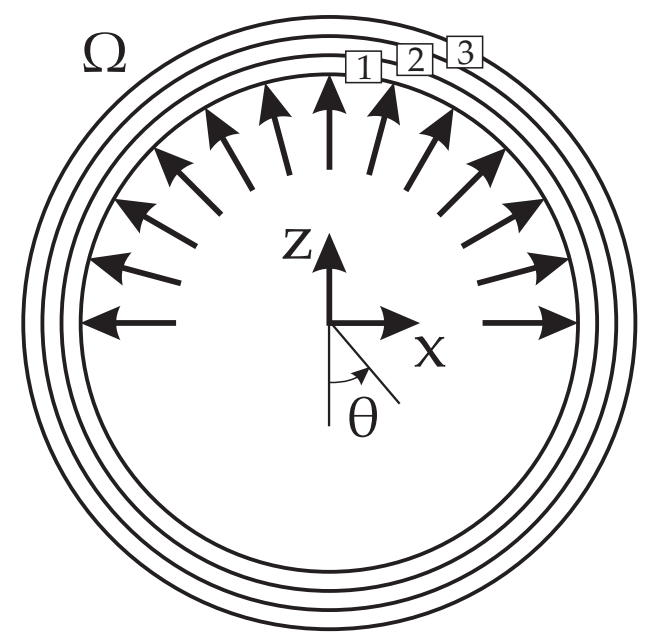

Figure 2. Pressure applied on the cylinder for the static analysis.

The static response of the structure is computed through the present CUF 1D model with a variable expansion order up to $N=8$ by solving Eq. 22. A solid finite element analysis is also carried out via the commercial code NASTRAN in order to assess the present refined 1D model. One solid element along the thickness is used for each layer. Due to the small layer thickness and the well-known aspect ratio restrictions typical of solid finite elements, the model in NASTRAN consists of 86880 nodes and 64800 HEX8 elements. The number of degrees of freedom (DOF) is thus equal to 257760 .

The deformation of the mid-span section, which is expressed in terms of the magnitude of displacement vector $\boldsymbol{u}$, is depicted in Fig. 3 for different models and compared with the solid NASTRAN solution. The solution confirms to be symmetrical with respect to the $x=0$ plane regardless the model used. The particular distribution of pressure loadings is supposed to deform the beam cross-section, in addition to a bending behavior obviously. Moreover, the span-to-external diameter ratio is equal to 5 and makes the structure a thick beam. Hence, this case cannot be consistent with the kinematic hypotheses which classical beam models are based on. Classical models are therefore not expected to yield accurate results and this statement is confirmed by the EBBM result. In fact, a pure bending and a uniform displacement over the cross-section are detected by Euler-Bernoulli theory.

By enriching the displacement field, the first-order model provide a linear displacement distribution along the $z$ direction. Nevertheless, it results not to be realistic. Taking the solid FE model as reference, the results improve as the expansion order $N$ increases. In particular, for a eight-order model the deformation of the mid-span section is accurately described and its agreement with the 3D solution is remarkable. Even not reported here, it is noteworthy that for 

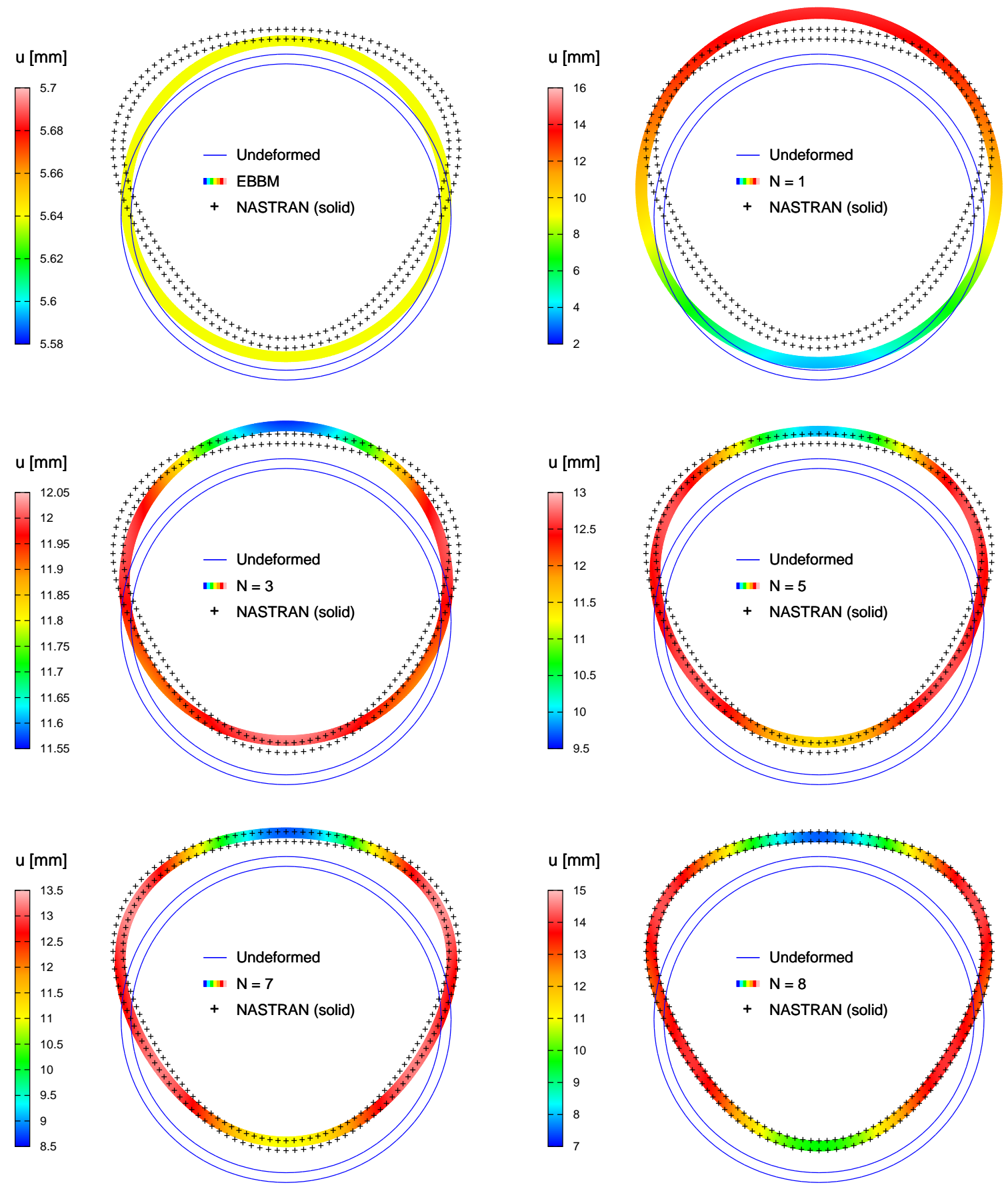

Figure 3. Deformation of the mid-span section for higher-order 1D models. 
an expansion order higher than 8 the analysis would provide the same results as those obtained by $N=8$, thus confirming a convergent trend on $N$.

The increasing accuracy of the present formulation as $N$ increases is well-shown in Fig. 4, which illustrates the deformation of the external edge of the annular mid-span section of the cylinder $\left(r=d_{e} / 2, y=L / 2\right)$ along the angular $\theta$ coordinate. The deformation is again expressed in terms of the magnitude of displacement vector $\boldsymbol{u}$. It is notable how the actual trend is far from the uniformly deformed configuration supposed by EBBM. Classical and low-order models provide a unrealistic behavior of the thin-walled cylinder. With a higher-order model than the third the curve trend changes and becomes similar to the reference one. Nevertheless, for $N<8$ the displacement is remarkably overestimated at points $\theta=180^{\circ}$ and dangerously underestimated at points where the displacement is actually maximum. The CUF 1D FEs provide a convergent solution by approaching the NASTRAN 3D results until a well agreement for $N=8$.

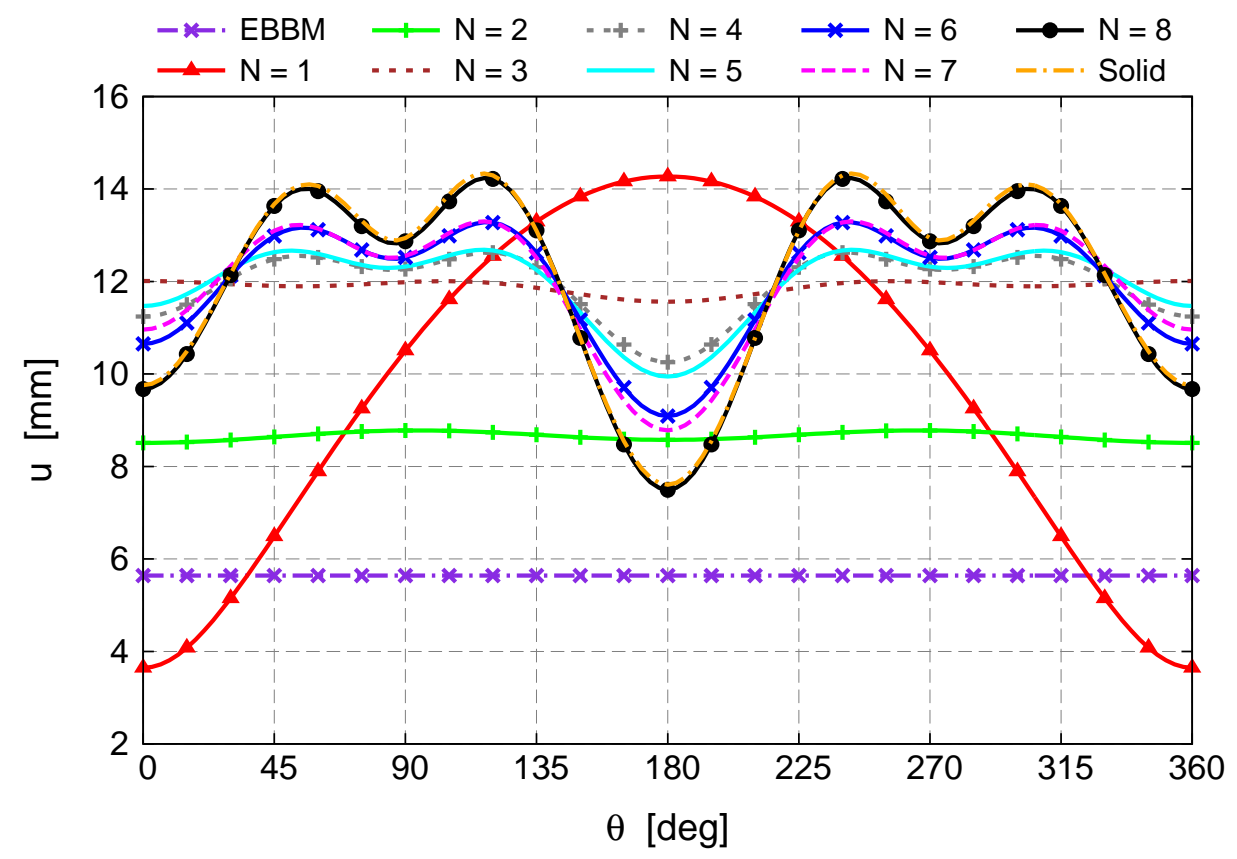

Figure 4. Displacement of the external edge of the mid-span section.

The maximum displacement on the external edge of the mid-span section is reported in Table 2. The error in computing the maximum deflection is significant for classical and low-order models, except for $N=1$. However, the solution for the first-order model is completely unrealistic, see Fig. 4. In general, $u_{\max }$ increases as $N$ increases approaching the $3 \mathrm{D}$ value. Also the position of the maximum displacement along the external edge in terms of $\theta$ appreciably changes for different models. $\theta_{u_{\max }}$ for 1D models with an expansion order higher than 4 coincides with the reference solution, whereas low-order models evidence their low accuracy even in the position evaluation. This aspect has not to be underestimate because it turns out to be fundamental for a failure investigation, for instance. In conclusion, the eight-order model proves its capability to exactly detect the three-dimensional deformation of the layered thin-walled cylinder with a sizeable reduction in computational cost in terms of DOF (4185 vs. 257760). 
Table 2. Maximum displacement $[\mathrm{mm}]$ on the external edge of the mid-span section.

\begin{tabular}{lrcrr}
\hline \hline Model & $u_{\max }$ & \% Difference & $\theta_{u_{\max }}$ & DOF \\
\hline \hline EBBM & 5.639 & -60.663 & - & 93 \\
$\mathrm{~N}=1$ & 14.272 & -0.439 & 180 & 279 \\
$\mathrm{~N}=2$ & 8.780 & -38.751 & 93 & 558 \\
$\mathrm{~N}=3$ & 12.011 & -16.212 & 0 & 930 \\
$\mathrm{~N}=4$ & 12.625 & -11.929 & 120 & 1395 \\
$\mathrm{~N}=5$ & 12.685 & -11.510 & 117 & 1953 \\
$\mathrm{~N}=6$ & 13.281 & -7.353 & 117 & 2604 \\
$\mathrm{~N}=7$ & 13.300 & -7.220 & 117 & 3348 \\
$\mathrm{~N}=8$ & 14.236 & -0.691 & 117 & 4185 \\
Solid FEM & 14.335 & - & 117 & 257760 \\
\hline
\end{tabular}

\subsection{Free vibration analysis}

The free vibration analysis of the same layered cylinder as that used for the previous static case is now faced by solving Eq. 23. The same solid FE model is also considered to carry out the free vibration analysis of the layered cylinder in NASTRAN (sol 103). All the vibrational modes obtained can be divided into four categories: bending, axial, radial, and lobes-type modes. In axial modes, the cylinder vibrates along its longitudinal axis $y$ and the cross-sections remain annular-type. In radial modes, the cross-sections vibrate radially remaining annular-type and rotate along the direction $\theta$. In lobes-type modes, the crosssections do not remain annular-type since two, theee, or four lobes and so on compare in the cross-sections.

Table 3 summarizes the natural frequencies computed for bending, radial, and axial vibrational modes through different models. As far as the bending modes are concerned, each frequency value refers to a couple of numbers, denoted as mode indexes, whose meaning is important to be explained. Let the vibrational modes computed by EBBM to be considered for instance. The first and second modes correspond to the same natural frequency (1963.0 $\mathrm{Hz}$ ) and represent the same way of vibrating, that is a single-wave bending mode. In fact, the cylinder is axisymmteric and thus can vibrate along a single-wave either in the $x-y$ plane or in the $z-y$ plane. Hence, the mode indexes related to EBBM single-wave bending mode $(1963.0 \mathrm{~Hz})$ are 1 and 2. According to Table 3, the third mode is axial whereas the fourth and fifth modes are double-wave bending modes and so on.

The first radial frequency is well-computed by all the models, even for $N=1$. This does not occur for bending modes, for which the expansion order has to be at least equal to 4 to match solid results with a minor error. Euler-Bernoulli theory is ineffective even in the computation of bending frequencies. Moreover, EBBM is unable to detect any radial mode due to the kinematic hypoteses it is based on. On the contrary, this model computes accurately the natural frequency of the first axial mode, even better than higher-order models. However, even for the axial mode the present formulation provides a convergent trend as $N$ increases.

Among all the possible lobe-types modes (with two, three, four lobes and so on), the 
Table 3. Natural frequencies $[\mathrm{Hz}]$ of bending, radial, and axial modes.

\begin{tabular}{lccccr}
\hline \hline \multirow{2}{*}{ Model } & \multicolumn{2}{c}{ Bending } & Radial & \multicolumn{1}{c}{ Axial } & \multirow{2}{*}{ DOF } \\
\cline { 2 - 4 } & \multicolumn{1}{c}{ Mode 1 } & Mode 2 & Mode 1 & Mode 1 & \\
\hline \hline \multirow{2}{*}{ EBBM } & $1963.0^{1,2}$ & $5044.9^{4,5}$ & - & $4173.2^{3}$ & 93 \\
$\mathrm{~N}=1$ & $1572.6^{1,2}$ & $3530.3^{6,7}$ & $2540.9^{4}$ & $4173.3^{8}$ & 279 \\
$\mathrm{~N}=2$ & $1597.7^{1,2}$ & $3562.1^{4,5}$ & $2540.9^{3}$ & $4191.3^{6}$ & 558 \\
$\mathrm{~N}=3$ & $1368.0^{1,2}$ & $2925.0^{6,7}$ & $2540.9^{5}$ & $4182.5^{10}$ & 930 \\
$\mathrm{~N}=4$ & $1365.4^{3,4}$ & $2912.6^{10,11}$ & $2540.9^{7}$ & $4182.4^{18}$ & 1395 \\
$\mathrm{~N}=5$ & $1364.3^{3,4}$ & $2909.7^{10,11}$ & $2540.9^{7}$ & $4182.1^{22}$ & 1953 \\
$\mathrm{~N}=6$ & $1364.3^{3,4}$ & $2909.6^{16,17}$ & $2540.9^{11}$ & $4182.0^{24}$ & 2604 \\
$\mathrm{~N}=7$ & $1364.2^{3,4}$ & $2909.5^{16,17}$ & $2540.9^{11}$ & $4182.0^{24}$ & 3348 \\
Solid FEM & $1360.9^{3,4}$ & $2906.3^{16,17}$ & $2540.2^{13}$ & $4172.2^{32}$ & 257760 \\
\hline
\end{tabular}

two-lobe frequencies computed by the present CUF 1D finite elements are considered and reported in Table 4. As can be seen, also the lobes-type modes have two mode indexes for each frequency, due to the cylinder axisymmetry. It is important to note that the increase of $N$ usually corresponds to a detection of new lobes-type modes. For instance, the second-order model is not able to detect any two-lobe mode. The third-order model is instead able to compute such modes, even if the corresponding frequencies are sizeable far from the correct frequencies. As a consequence, it is necessary to increase the expansion order to 7 to match the NASTRAN reference results, see Table 4. In the same way, for instance the four-lobe modes are not detectable for an expansion order lower than 6. This is the reason why the increase of the accuracy of the 1D model improves not also the computation of the frequency values, but also the corresponding mode indexes.

Table 4. Natural frequencies [Hz] of two-lobe modes.

\begin{tabular}{lrrrr}
\hline \hline \multirow{2}{*}{ Model } & \multicolumn{3}{c}{ Mode number } & \multirow{2}{*}{ DOF } \\
\cline { 2 - 3 } & \multicolumn{1}{c}{1} & \multicolumn{1}{c}{2} & \multicolumn{1}{c}{3} \\
\hline \hline $\mathrm{N}=3$ & $1617.9^{3,4}$ & $2960.7^{8,9}$ & $4755.5^{13,14}$ & 930 \\
$\mathrm{~N}=4$ & $1209.1^{1,2}$ & $1795.0^{5,6}$ & $2732.8^{8,9}$ & 1395 \\
$\mathrm{~N}=5$ & $1005.4^{1,2}$ & $1666.3^{5,6}$ & $2643.3^{8,9}$ & 1953 \\
$\mathrm{~N}=6$ & $995.8^{1,2}$ & $1640.9^{5,6}$ & $2602.6^{12,13}$ & 2604 \\
$\mathrm{~N}=7$ & $862.7^{1,2}$ & $1561.9^{5,6}$ & $2551.7^{12,13}$ & 3348 \\
Solid FEM & $859.9^{1,2}$ & $1557.0^{5,6}$ & $2545.4^{14,15}$ & 257760 \\
\hline
\end{tabular}

It is important to note that the lobes-type modes are typically not detectable by any onedimensional model. In fact, shell (2D) or solid (3D) models need to be usually involved to study thin-walled structures such as the cylinder here considered. Pointing out the agreement of $N=7$ frequencies with NASTRAN frequencies, the results demonstrate the shell-type capabilities of the present $1 \mathrm{D}$ FEs in accurately predicting complex vibrational modes and the 
corresponding frequency values with a striking reduction of DOF. For the sake of brevity, further numerical comparisons on complex vibrational modes such as third- and fourth-lobe modes are not reported here. However, as an example case the sixth three-lobe mode computed by the present CUF 1D model is depicted in Fig. 5 and graphically compared with the corresponding mode obtained by NASTRAN solid model, see Fig. 6 . The vibrational modes perfectly match.

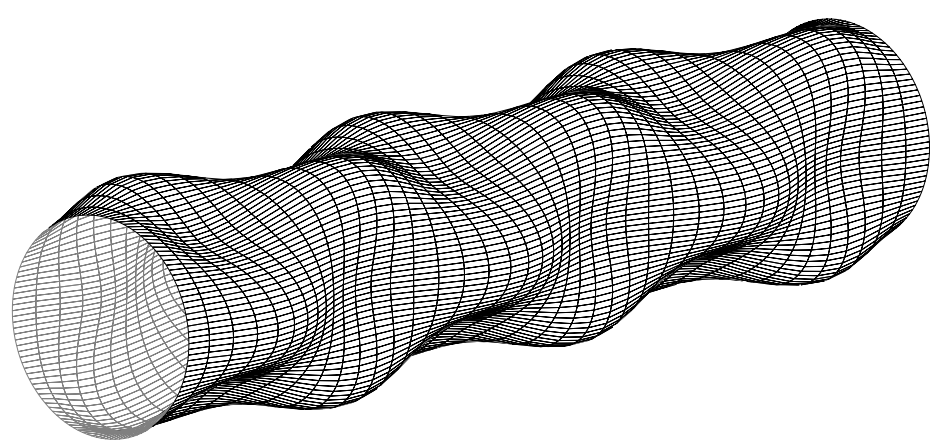

Figure 5. Sixth three-lobe mode. Present CUF 1D.

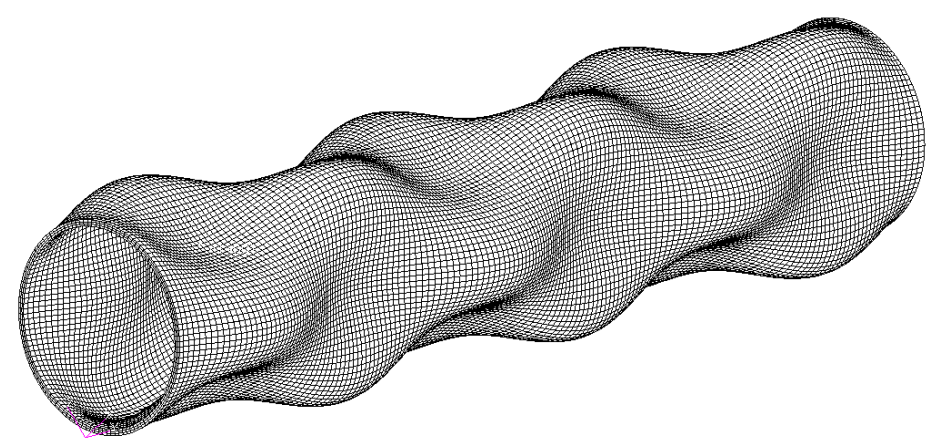

Figure 6. Sixth three-lobe mode. NASTRAN solid.

\section{CONCLUSIONS}

This paper presents the application of refined one-dimensional models to the static and free vibration analysis of thin-walled structures. The 1D Carrera Unified Formulation, CUF, is exploited to formulate higher-order 1D finite elements with variable kinematics whose form does not depend on the expansion order chosen.

A layered cylinder were used to assess and enhance the advantages of refined 1D CUF models in evaluating the behavior of thin-walled structures. The effectiveness of higher-order terms over the cross-section deformation were highlighted mainly when thin-walled geometries are adopted. In fact for these cases classical beam theories become ineffective, since they assume an undeformed cross-section. Comparing results with solid (3D) FE solutions, in-plane deformations are instead accurately described by the proposed 1D model by increasing the order of expansion and involving a limited number of degrees of freedom.

The dynamic analysis of the structure revealed the shell-type capabilities of the present 1D model by detecting vibrational modes typical of thin-walled structures and usually detectable 
by shell or solid models. The study of corresponding natural frequencies showed a well agreement with $3 \mathrm{D}$ results with a remarkable reduction in computational cost.

In conclusion, the present $1 \mathrm{D}$ finite element formulation proved to be a valid alternative to shell and solid methods which necessarily require a higher computational cost. Future works could address the structural dynamics of complex multilayered composite structures for typical aerospace and biomechanical applications.

\section{REFERENCES}

[1] Bathe K., Finite element procedure. New Jersey: Prentice Hall, Upper Saddle River, 1996.

[2] Bickford W. B., "A consistent higher-order beam theory", Developments in Theoretical and Applied Mechanics, vol. 11, pp. 137-142, 1982.

[3] Carrera E., "Theories and finite elements for multilayered, anisotropic, composite plates and shells", Archives of Computational Methods in Engineering, vol. 9, no. 2, pp. 87-140, 2002.

[4] Carrera E. and Brischetto S., "Analysis of thickness locking in classical, refined and mixed multilayered plate theories", Composite Structures, vol. 82, no. 4, pp. 549-562, 2008.

[5] Carrera E. and Brischetto S., "Analysis of thickness locking in classical, refined and mixed theories for layered shells", Composite Structures, vol. 85, no. 1, pp. 83-90, 2008.

[6] Carrera E., Brischetto S., and Nali P., Plates and Shells for Smart Structures: Classical and Advanced Theories for Modeling and Analysis. John Wiley \& Sons, 2011.

[7] Carrera E. and Giunta G., "Refined beam theories based on a unified formulation", International Journal of Applied Mechanics, vol. 2, no. 1, pp. 117-143, 2010.

[8] Carrera E., Giunta G., and Petrolo M., Beam Structures: Classical and Advanced Theories. John Wiley \& Sons, 2011.

[9] Carrera E., Giunta G., Nali P., and Petrolo M., "Refined beam elements with arbitrary cross-section geometries", Computers and Structures, vol. 88, no. 5-6, pp. 283-293, 2010 .

[10] Carrera E. and Petrolo M., "Refined one-dimensional formulations for laminated structure analysis", AIAA Journal, 2012. In press, DOI: 10.2514/1.J051219.

[11] Carrera E., Petrolo M., and Nali P., "Unified formulation applied to free vibrations finite element analysis of beams with arbitrary section", Shock and Vibrations, vol. 18, no. 3, pp. 485-502, 2010.

[12] Carrera E., Petrolo M., and Varello A., "Advanced beam formulations for free vibration analysis of conventional and joined wings", Journal of Aerospace Engineering, 2012. In press, DOI: 10.1061/(ASCE)AS.1943-5525.0000130.

[13] Euler L., De curvis elasticis. Lausanne and Geneva: Bousquet, 1744.

[14] Ganesan R. and Zabihollah A., "Vibration analysis of tapered composite beams using a higher-order finite element, Part I: Formulation”, Composite Structures, vol. 77, no. 3, pp. 306-318, 2007. 
[15] Ganesan R. and Zabihollah A., "Vibration analysis of tapered composite beams using a higher-order finite element, Part II: Parametric study", Composite Structures, vol. 77, no. 3, pp. 319-330, 2007.

[16] Heyliger P. R. and Reddy J. N., "A higher order beam finite element for bending and vibration problems", Journal of Sound and Vibration, vol. 126, no. 2, pp. 309-326, 1988.

[17] Jones R., Mechanics of Composite Materials. Philadelphia: Taylor \& Francis, 1999.

[18] Kant T. and Gupta A., "A finite element model for a higher-order shear deformable beam theory”, Journal of Sound and Vibration, vol. 125, no. 2, pp. 193-202, 1988.

[19] Kant T., Marur S. R., and Rao G.S., "Analytical solution to the dynamic analysis of laminated beams using higher order refined theory", Composite Structures, vol. 40, no. 1, pp. 1-9, 1997.

[20] Kapania K. and Raciti S., "Recent advances in analysis of laminated beams and plates, Part II: Vibrations and wave propagation”, AIAA Journal, vol. 27, no. 7, pp. 935-946, 1989.

[21] Levinson M., "A new rectangular beam theory”, Journal of Sound and Vibration, vol. 174, no. 1, pp. 81-87, 1981.

[22] Marur S. R. and Kant T., "Free vibration analysis of fiber reinforced composite beams using higher order theories and finite element modelling", Journal of Sound and Vibration, vol. 194, no. 3, pp. 337-351, 1996.

[23] Marur S. R. and Kant T., "On the angle ply higher order beam vibrations", Computational Mechanics, vol. 40, pp. 25-33, 2007.

[24] Rychter Z., "On the accuracy of a beam theory", Mechanical Research Communications, vol. 14, no. 2, pp. 99-105, 1987.

[25] Silvestre N., "Second-order generalised beam theory for arbitrary orthotropic materials", Thin-Walled Structures, vol. 40, no. 9, pp. 791-820, 2002.

[26] Şimşek M. and Kocatürk T., "Free vibration analysis of beams by using a third-order shear deformation theory”, Sädhanā, vol. 32, no. 3, pp. 167-179, 2007.

[27] Soldatos K. P. and Elishakoff I., "A transverse shear and normal deformable orthotropic beam theory", Journal of Sound and Vibration, vol. 155, no. 3, pp. 528-533, 1992.

[28] Stephen N. G. and Levinson M., "A second order beam theory”, Journal of Sound and Vibration, vol. 67, no. 3, pp. 293-305, 1979.

[29] Subramanian P., "Dynamic analysis of laminated composite beams using higher order theories and finite elements", Composite Structures, vol. 73, no. 3, pp. 342-353, 2006.

[30] Timoshenko S., "On the correction for shear of the differential equation for transverse vibrations of prismatic bars", Philosophical Magazine, vol. 41, pp. 744-746, 1921.

[31] Varello A., Petrolo M., and Carrera E., "A refined 1D FE model for the application to aeroelasticty of composite wings", in IV International Conference on Computational Methods for Coupled Problems in Science and Engineering, (Kos Island, Greece), June 2011.

[32] Yu W., Volovoi V., Hodges D., and Hong X., "Validation of the variational asymptotic beam sectional analysis (VABS)", AIAA Journal, vol. 40, no. 10, pp. 2105-2113, 2002. 